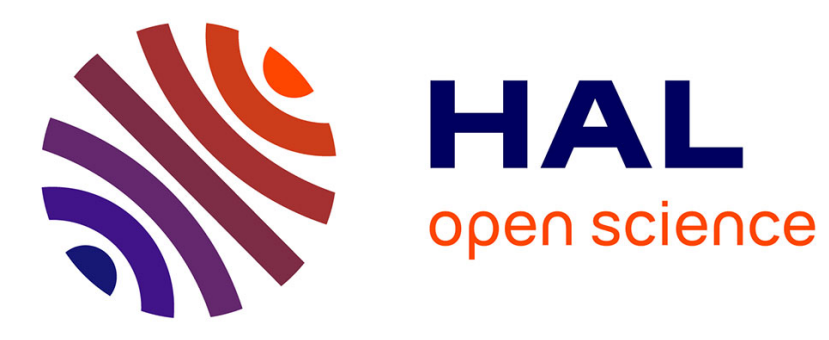

\title{
Decoherence time and spin measurement in the Stern-Gerlach experiment
}

\author{
Michel Gondran, Alexandre Gondran, Abdel Kenoufi
}

\section{To cite this version:}

Michel Gondran, Alexandre Gondran, Abdel Kenoufi. Decoherence time and spin measurement in the Stern-Gerlach experiment. AIP 2011, American Institue of Physics Conference on General Problems of Quantum Mechanics, Jun 2011, Växjö, Sweden. pp 116-120, 10.1063/1.3688960 . hal-00938521

\section{HAL Id: hal-00938521 \\ https://hal-enac.archives-ouvertes.fr/hal-00938521}

Submitted on 16 May 2014

HAL is a multi-disciplinary open access archive for the deposit and dissemination of scientific research documents, whether they are published or not. The documents may come from teaching and research institutions in France or abroad, or from public or private research centers.
L'archive ouverte pluridisciplinaire HAL, est destinée au dépôt et à la diffusion de documents scientifiques de niveau recherche, publiés ou non, émanant des établissements d'enseignement et de recherche français ou étrangers, des laboratoires publics ou privés. 


\title{
Decoherence time and spin measurement in the Stern-Gerlach experiment
}

\author{
Michel Gondran*, Alexandre Gondran ${ }^{\dagger}$ and Abdel Kenouf** \\ *University Paris Dauphine, 75016 Paris, France \\ ${ }^{\dagger}$ Ecole Nationale de l'Aviation Civile, 31000 Toulouse, France \\ ** SCORE, Scientific Consulting for Research and Engineering, 67000 Strasbourg, France
}

\begin{abstract}
We present an analytical solution to the decoherence time for the spin measurement and the diagonalization of the density matrix of spin variables in the Stern-Gerlach experiment. This solution requires the calculation of the Pauli spinor with a spatial extension, which is not found in quantum mechanics textbooks. With this full spinor and the measured position of the particle we demonstrate the three postulates of quantum measurement: quantization, spectral decomposition and wave function reduction. The transition from a quantum superposition to a statistical mixture is well explained in this way, but not the single result that always emerges from a particular experiment. The spinor spatial extension also allows the introduction of the de Broglie-Bohm trajectories which give a very simple explanation of the particles' impact.
\end{abstract}

Keywords: Stern and Gerlach, decoherence, postulates of quantum measurement,Pauli spinor,density matrix

PACS: 03.65.Ta, 03.65.Yz

\section{INTRODUCTION}

In 1922, by studying the deflection of a beam of silver atoms in a strongly inhomogeneous magnetic field (cf. FIG. 1) Otto Stern and Walter Gerlach [1] obtained an experimental result that contradicts the common sense prediction: the beam, instead of expanding, splits into two separate beams giving two spots of equal intensity $N^{+}$and $N^{-}$on a detector, at equal distances from the axis of the original beam. Historically, this is the experiment which helped establish the spin quantization. Theoretically, it is the seminal experiment posing the problem of measurement in quantum mechanics. Today it is the theory of decoherence with the diagonalization of the density matrix that is put forward to explain the first part of the measurement process $[2,3,4,5]$.

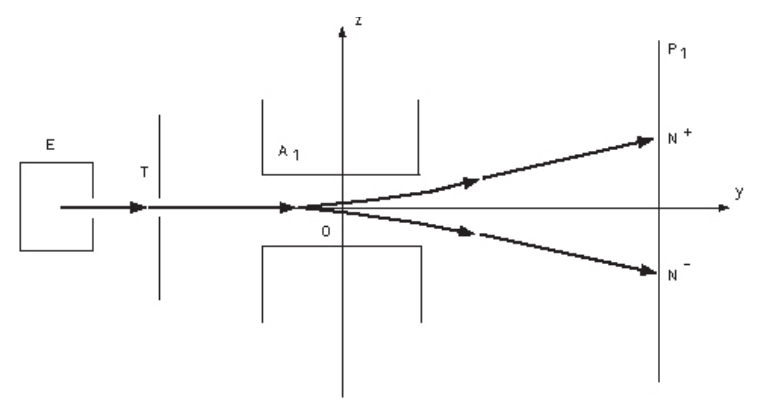

FIGURE 1. Schematic configuration of the Stern-Gerlach experiment.

Foundations of Probability and Physics - 6 AIP Conf. Proc. 1424, 116-120 (2012); doi: 10.1063/1.3688960 (C) 2012 American Institute of Physics 978-0-7354-1004-6/\$30.00 
However, although these authors consider the Stern-Gerlach experiment as fundamental, they do not propose a numerical simulation of the measurement process or a calculation of the spin decoherence time.

The aim of this paper is to present an analytical solution to this decoherence time and the diagonalization of the density matrix. This solution requires the calculation of the Pauli spinor with a spacial extension as the equation:

$$
\Psi^{0}(z)=\left(2 \pi \sigma_{0}^{2}\right)^{-\frac{1}{2}} e^{-\frac{z^{2}}{4 \sigma_{0}^{2}}}\left(\begin{array}{c}
\cos \frac{\theta_{0}}{2} e^{-i \frac{\varphi_{0}}{2}} \\
\sin \frac{\theta_{0}}{2} e^{i \frac{\varphi_{0}}{2}}
\end{array}\right) .
$$

Quantum mechanics textbooks $[6,7,8,9]$ do not take into account the spatial extension of the spinor (1) and simply use the simplified spinor without spatial extension:

$$
\Psi^{0}=\left(\begin{array}{c}
\cos \frac{\theta_{0}}{2} e^{-i \frac{\varphi_{0}}{2}} \\
\sin \frac{\theta_{0}}{2} e^{i \frac{\varphi_{0}}{2}}
\end{array}\right) .
$$

However, as we shall see, the different evolution of the spatial extension between the two spinor components will have a key role in the explanation of the measurement process. This spatial extension enables us, in following the precursory works of Takabayasi [10], Bohm [11, 12] and Dewdney et al. [13], to revisit the Stern and Gerlach experiment, to explain the decoherence and to demonstrate the three postulates of the measure: quantization, spectral decomposition and wave function reduction.

\section{THE STERN-GERLACH EXPERIMENT}

Silver atoms contained in the oven E (Fig. 1) are heated to a high temperature and escape through a narrow opening. A second aperture, T, selects those atoms whose velocity, $\mathbf{v}_{0}$, is parallel to the y-axis. The atomic beam crosses the gap of the electromagnet $A_{1}$ before condensing on the detector, $P_{1}$. Before crossing the electromagnet, the magnetic moments of the silver atoms are oriented randomly (isotropically). In the beam, we represent the atoms by their wave function; one can assume that at the entrance to the electromagnet, $A_{1}$, and at the initial time $t=0$, each atom can be approximatively described by a Gaussian spinor in $\mathrm{z}$ given by (1) corresponding to a pure state. The variable y will be treated classically with $y=v t . \sigma_{0}=10^{-4} \mathrm{~m}$ corresponds to the size of the slot $\mathrm{T}$ following Oz. The approximation by a Gaussian initial spinor will allow explicit calculations. Because the slot is much wider following $\mathrm{Ox}$, the variable $\mathrm{x}$ will be also treated classically. For the silver atom, we have $m=1.8 \times 10^{-25} \mathrm{~kg}, v_{0}=500$ $\mathrm{m} / \mathrm{s}$ (with $\mathrm{T}=1000 \mathrm{~K}$ ). In (1), $\theta_{0}$ and $\varphi_{0}$ are the polar angles characterizing the initial orientation of the magnetic moment, $\theta_{0}$ corresponds to the angle with the z-axis. The experiment is a statistical mixture of pure states where the $\theta_{0}$ and the $\varphi_{0}$ are randomly chosen: $\theta_{0}$ is drawn in a uniform way from $[0, \pi]$ and that $\varphi_{0}$ is drawn in a uniform way from $[0,2 \pi]$. 
The evolution of the spinor $\Psi=\left(\begin{array}{c}\psi_{+} \\ \psi_{-}\end{array}\right)$in a magnetic field $\mathbf{B}$ is then given by the Pauli equation:

$$
i \hbar\left(\begin{array}{c}
\frac{\partial \psi_{+}}{\partial t} \\
\frac{\partial \psi_{-}}{\partial t}
\end{array}\right)=-\frac{\hbar^{2}}{2 m} \Delta\left(\begin{array}{l}
\psi_{+} \\
\psi_{-}
\end{array}\right)+\mu_{B} \mathbf{B} \sigma\left(\begin{array}{l}
\psi_{+} \\
\psi_{-}
\end{array}\right)
$$

where $\mu_{B}=\frac{e \hbar}{2 m_{e}}$ is the Bohr magneton and where $\sigma=\left(\sigma_{x}, \sigma_{y}, \sigma_{z}\right)$ corresponds to the three Pauli matrixes. The particle first enters an electromagnetic field $\mathbf{B}$ directed along the $z$-axis, $B_{x}=B_{0}^{\prime} x, B_{y}=0, B_{z}=B_{0}-B_{0}^{\prime} z$, with $B_{0}=5$ Tesla, $B_{0}^{\prime}=\left|\frac{\partial B}{\partial z}\right|=10^{3} \mathrm{Tesla} / \mathrm{m}$ over a length $\Delta l=1 \mathrm{~cm}$. On exiting the magnetic field, the particle is free until it reaches the detector $P_{1}$ placed at a $D=20 \mathrm{~cm}$ distance.

The particle stays within the magnetic field for a time $\Delta t=\frac{\Delta l}{v}=2 \times 10^{-5} \mathrm{~s}$. After the magnetic field, at time $t+\Delta t(t \geq 0)$ in the free space, the spinor equals $[12,13,14,15]$ :

$$
\Psi(z, t+\Delta t) \simeq\left(\begin{array}{c}
\cos \frac{\theta_{0}}{2}\left(2 \pi \sigma_{0}^{2}\right)^{-\frac{1}{2}} e^{-\frac{\left(z-z_{\Delta}-u t\right)^{2}}{4 \sigma_{0}^{2}}} e^{i \frac{m u z+\hbar \varphi_{+}}{\hbar}} \\
\sin \frac{\theta_{0}}{2}\left(2 \pi \sigma_{0}^{2}\right)^{-\frac{1}{2}} e^{-\frac{\left(z+z_{\Delta}+u t\right)^{2}}{4 \sigma_{0}^{2}}} e^{i \frac{-m u z+\hbar \varphi_{-}}{\hbar}}
\end{array}\right)
$$

where

$$
z_{\Delta}=\frac{\mu_{B} B_{0}^{\prime}(\Delta t)^{2}}{2 m}=10^{-5} m, \quad u=\frac{\mu_{B} B_{0}^{\prime}(\Delta t)}{m}=1 \mathrm{~m} / \mathrm{s} .
$$

Equation (4) takes into account the spatial extension of the spinor and we note that the two spinor components have very different $z$ values. All interpretations of this paper are based on this equation.

\section{THE DECOHERENCE TIME}

We deduce from 4) the probability density of a pure state in the free space after the electromagnet:

$$
\rho_{\theta_{0}}(z, t+\Delta t) \simeq\left(2 \pi \sigma_{0}^{2}\right)^{-\frac{1}{2}}\left(\cos ^{2} \frac{\theta_{0}}{2} e^{-\frac{(z-z \Delta-u t)^{2}}{2 \sigma_{0}^{2}}}+\sin ^{2} \frac{\theta_{0}}{2} e^{-\frac{\left(z+z_{\Delta}+u t\right)^{2}}{2 \sigma_{0}^{2}}}\right)
$$

Figure 2 shows the probability density of a pure state (with $\theta_{0}=\pi / 3$ ) as a function of $\mathrm{z}$ at several values of $\mathrm{t}$ (the plots are labelled $y=v t$ ). The beam separation does not appear at the end of the magnetic field $(1 \mathrm{~cm})$, but $16 \mathrm{~cm}$ further along. It is the moment of the decoherence. The decoherence time, where the two spots $N^{+}$and $N^{-}$are separated, is then given by the equation:

$$
t_{D} \simeq \frac{3 \sigma_{0}-z_{\Delta}}{u}=\frac{\left(3 \sigma_{0}-z_{\Delta}\right) m v}{\mu_{B} B_{0}^{\prime} \Delta l}=3 \times 10^{-4} s .
$$



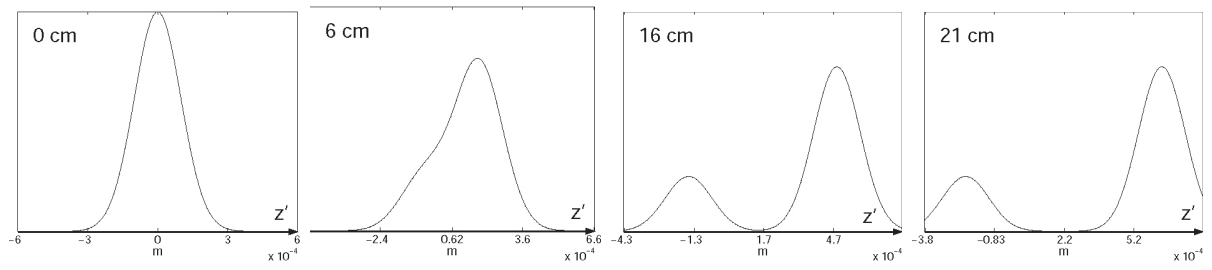

FIGURE 2. Evolution of the probability density of a pure state with $\theta_{0}=\pi / 3$.

This decoherence time is usually the time required to diagonalize the marginal density matrix of spin variables associated with a pure state [16]:

$$
\rho^{S}(t)=\left(\begin{array}{cc}
\int\left|\psi_{+}(z, t)\right|^{2} d z & \int \psi_{+}(z, t) \psi_{-}^{*}(z, t) d z \\
\int \psi_{-}(z, t) \psi_{+}^{*}(z, t) d z & \int\left|\psi_{-}(z, t)\right|^{2} d z
\end{array}\right)
$$

For $t \geq t_{D}$, the product $\psi_{+}(z, t+\Delta t) \psi_{-}(z, t+\Delta t)$ is null and the density matrix is diagonal: the probability density of the initial pure state (4) is diagonal:

$$
\rho^{S}(t+\Delta t)=\left(2 \pi \sigma_{0}^{2}\right)^{-1}\left(\begin{array}{cc}
\cos ^{2} \frac{\theta_{0}}{2} & 0 \\
0 & \sin ^{2} \frac{\theta_{0}}{2}
\end{array}\right)
$$

\section{PROOF OF THE POSTULATES OF QUANTUM MEASUREMENT}

We then obtain atoms with a spin oriented only along $\mathrm{z}$ (positively or negatively). Let us consider the spinor $\Psi(z, t+\Delta t)$ given by equation (4). Experimentally, we do not measure the spin directly, but the $\widetilde{z}$ position of the particle impact on $P_{1}$. If $\widetilde{z} \in N^{+}$, the term $\psi_{-}$of (4) is equal to zero and the spinor $\Psi$ is proportional to $\left(\begin{array}{l}1 \\ 0\end{array}\right)$, one of the eigenvectors of $\sigma_{z}$ :

$$
\Psi(\tilde{z}, t+\Delta t) \simeq\left(2 \pi \sigma_{0}^{2}\right)^{-\frac{1}{4}} \cos \frac{\theta_{0}}{2} e^{-\frac{\left(\tilde{z}_{1}-z_{\Delta}-u t\right)^{2}}{4 \sigma_{0}^{2}}} e^{i \frac{m u \tilde{z}_{1}+\hbar \varphi_{+}}{\hbar}}\left(\begin{array}{l}
1 \\
0
\end{array}\right) .
$$

If $\widetilde{z} \in N^{-}$, the term $\psi_{+}$of (4) is equal to zero and the spinor $\Psi$ is proportional to $\left(\begin{array}{l}0 \\ 1\end{array}\right)$, the other eigenvector of $\sigma_{z}$ :

$$
\Psi(\tilde{z}, t+\Delta t) \simeq\left(2 \pi \sigma_{0}^{2}\right)^{-\frac{1}{4}} \sin \frac{\theta_{0}}{2} e^{-\frac{\left(\tilde{z}_{2}+z_{\Delta}+u t\right)^{2}}{4 \sigma_{0}^{2}}} e^{i \frac{-m u \tilde{z}_{2}+\hbar \varphi_{-}}{\hbar}}\left(\begin{array}{l}
0 \\
1
\end{array}\right) .
$$

Therefore, the measurement of the spin corresponds to an eigenvalue of the spin operator $S_{z}=\frac{\hbar}{2} \sigma_{z}$. It is a proof of the postulate of quantization.

Equation (9) gives the probability $\cos ^{2} \frac{\theta_{0}}{2}\left(\right.$ resp. $\left.\sin ^{2} \frac{\theta_{0}}{2}\right)$ to measure the particle in the spin state $+\frac{\hbar}{2}$ (resp. $-\frac{\hbar}{2}$ ); this proves the spectral decomposition postulate.

By drilling a hole in the detector $P_{1}$ to the location of the spot $N^{+}$(Fig.1), we select the atoms that are all in the spin state $|+\rangle=\left(\begin{array}{l}1 \\ 0\end{array}\right)$. The new spinor of this atoms is obtained by making the component $\Psi_{-}$of the spinor $\Psi$ identically zero at the time when the atom 
crosses the detector $P_{1}$; at this time the component $\Psi_{-}$is indeed stopped by detector $P_{1}$. The future of the silver atom after crossing the detector $P_{1}$ will be guided by this new (normalized) spinor. The reduction of the wave function is therefore not linked to the electromagnet, but to the detector $P_{1}$ causing an irreversible elimination of the spinor component $\Psi_{-}$.

Finally, it remains to provide an explanation of the individual impacts of silver atoms. The spatial extension of the spinor (1) allows to take into account the particle's initial position $z_{0}$ and to introduce the Broglie-Bohm trajectories [13, 14, 17, 18, 19] which are the natural assumption to explain the individual impacts.

\section{CONCLUSION}

We conclude that the resolution in space and time of the Pauli equation is essential to theoretically obtain spatial quantization and decoherence time in the Stern-Gerlach experiment. The joint use of a measured position and a spatial spinor enables us to demonstrate the three quantum measurement postulates. The de Broglie-Bohm trajectories are then used to provide a natural explanation to the particles' impacts. The result of the Stern-Gerlach experiment is not the measure of the spin projection along the z-axis, but the orientation of the spin either in the direction of the magnetic field gradient, or in the opposite direction. It depends on the position of the particle in the wave function. We have therefore a simple explanation for the non-compatibility of spin measurements along different axes.

\section{REFERENCES}

1. W. Gerlach and O. Stern, Zeit. Phys. 8, 110 (1921); Zeit. Phys. 9, 349 (1922).

2. H. D. Zeh, Found. Phys.,1, 69 (1970).

3. W. H. Zurek, Phys.Rev. D26 1862 (1982) ; J.A. Wheeler, and W. H. Zurek, Quantum Theory of Measurement (Princeton University Press, 1983); W. H. Zurek, Rev. Mod. Phys. 75 (2003) 715.

4. R. Omnes, Rev. Mod. Phys. 64, 339 (1992).

5. M. Schlosshauer, Decoherence and the Quantum-to-Classical Transition (Springer-Verlag, 2007).

6. R. P. Feynman, R. B. Leighton, and M. Sands, The Feynman Lectures on Physics (Addison-Wesley, New York, 1965).

7. C. Cohen-Tannoudji, B. Diu, and F. Laloë, Quantum Mechanics (Wiley, New York, 1977).

8. J.J. Sakurai, Modern Quantum Mechanics (Addison-Wesley, 1985).

9. M. Le Bellac, Quantum Physics (Cambridge University Press, 2006).

10. T.Takabayasi, Prog. Theor. Phys., 8 2, 143 (1952); Prog. Theor. Phys., 11 4-5, 341 (1954).

11. D. Bohm, R. Schiller,and J. Tiomno, Nuovo Cim. supp. 1, 48-66 and 67-91(1955).

12. D. Bohm, and B.J. Hiley, The Undivided Universe (Routledge, London and New York, 1993).

13. C. Dewdney, P.R. Holland, and A. Kypianidis, Phys. Lett. A, 119(6), 259-267 (1986).

14. P.R. Holland, The Quantum Theory of Motion (Cambridge University Press, 1993).

15. M.Gondran, and A. Gondran, quant-ph/0511276.

16. G.B. Roston, M. Casas, A. Plastino and A.R. Plastino, Eur.J.Phys. 26 (2005) 657-672.

17. L. de Broglie, J. de Phys. 8, 225-241 (1927); Une tentative d'interprétation causale et non linéaire de la mécanique ondulatoire (Gauthier-Villars, Paris, 1951).

18. D. Bohm, Physical Review, 85, 166-193 (1952).

19. A. Challinor, A. Lasenby, S. Gull, and Chris Doran, Phys. Lett. A 218, 128-138 (1996). 